\title{
Long-range RNA pairings contribute to mutually exclusive splicing
}

\author{
YUAN YUE, ${ }^{1,3}$ YUN YANG, ${ }^{1,3}$ LANZHI DAI, ${ }^{1}$ GUOZHENG CAO, ${ }^{1}$ RAN CHEN, ${ }^{1}$ WEILING HONG, ${ }^{1}$ BAOPING LIU, ${ }^{1}$ \\ YANG SHI, ${ }^{1}$ YIJUN MENG, ${ }^{1}$ FENG SHI, ${ }^{1}$ MU XIAO, ${ }^{2}$ and YONGFENG JIN ${ }^{1}$ \\ ${ }^{1}$ Institute of Biochemistry, College of Life Sciences, Zhejiang University (Zijingang Campus), Hangzhou, Zhejiang, ZJ310058, China \\ ${ }^{2}$ Institute of Life Sciences, Zhejiang University (Zijingang Campus), Hangzhou, Zhejiang, ZJ310058, China
}

\begin{abstract}
Mutually exclusive splicing is an important means of increasing the protein repertoire, by which the Down's syndrome cell adhesion molecule (Dscam) gene potentially generates 38,016 different isoforms in Drosophila melanogaster. However, the regulatory mechanisms remain obscure due to the complexity of the Dscam exon cluster. Here, we reveal a molecular model for the regulation of the mutually exclusive splicing of the serpent pre-mRNA based on competition between upstream and downstream RNA pairings. Such dual RNA pairings confer fine tuning of the inclusion of alternative exons. Moreover, we demonstrate that the splicing outcome of alternative exons is mediated in relative pairing strength-correlated mode. Combined comparative genomics analysis and experimental evidence revealed similar bidirectional structural architectures in exon clusters 4 and 9 of the Dscam gene. Our findings provide a novel mechanistic framework for the regulation of mutually exclusive splicing and may offer potentially applicable insights into long-range RNA-RNA interactions in gene regulatory networks.
\end{abstract}

Keywords: mutually exclusive splicing; bidirectional RNA pairing; serpent; Dscam; regulatory mechanism

\section{INTRODUCTION}

Alternative splicing is a crucial source of proteome diversity, which plays an important role in many cellular processes and functions (Black 2003; Matlin et al. 2005; Blencowe 2006; Chen and Manley 2009; Nilsen and Graveley 2010). In humans, $\sim 95 \%$ of multiexon genes are alternatively spliced (Pan et al. 2008; Sultan et al. 2008), and these splicing events have implications for disease, since some mutations that influence the splicing process might cause genetic disorders. Alternative splicing includes various splicing events, such as exon skipping, alternative $5^{\prime}$ or $3^{\prime}$ splice site usage, intron retention, and mutually exclusive splicing (Black 2003; Matlin et al. 2005; Blencowe 2006; Nilsen and Graveley 2010). Mutually exclusive splicing is defined as a cluster of internal exons in which only one of the exons is included in a given mRNA (Smith 2005). The most extraordinary example of this is the arthropod Dscam (Down's syndrome cell adhesion molecule) pre-mRNA, which potentially generates 38,016 different mRNA isoforms in Drosophila melanogaster (Schmucker et al. 2000). In this case, the 95 alternative exons are organized into exon 4, 6, 9 and 17 clusters, containing 12, 48,33 , and 2 variable exons, respectively. The elaboration of

\footnotetext{
${ }^{3}$ These authors contributed equally to this work.

Corresponding author: jinyf@zju.edu.cn

Article published online ahead of print. Article and publication date are at http://www.rnajournal.org/cgi/doi/10.1261/rna.053314.115.
}

the Dscam locus in arthropod to produce large isoform repertoires with diverse expression patterns presents Dscam as a key target that could affect dramatic changes in adaptive immune or nervous responses (Watson et al. 2005; Chen et al. 2006). Indeed, much evidence has revealed that the extraordinary diversity of the Dscam isoform is required for its nervous and immune function (Zhan et al. 2004; Watson et al. 2005; Chen et al. 2006; Hattori et al. 2007, 2009; Matthews et al. 2007; Watthanasurorot et al. 2011; Dong et al. 2012; Miura et al. 2013; He et al. 2014).

Cis-acting RNA structures between docking site and selector sequence interactions were proposed to ensure that only one variant exon of Dscam is selected by the spliceosome (Graveley 2005; Anastassiou et al. 2006; Yang et al. 2011). This mechanism was initially discovered within the exon 6 cluster of Dscam, where the docking site in the intron downstream from constitutive exon 5 could pair with the selector sequences upstream of each of the 48 exon 6 variants (Graveley 2005). Combined with alternative RNA structures, the heterogeneous nuclear ribonucleoprotein hrp36 was shown to contribute to splicing fidelity (Olson et al. 2007). We

(C) 2015 Yue et al. This article is distributed exclusively by the RNA Society for the first 12 months after the full-issue publication date (see http:// rnajournal.cshlp.org/site/misc/terms.xhtml). After 12 months, it is available under a Creative Commons License (Attribution-NonCommercial 4.0 International), as described at http://creativecommons.org/licenses/by-nc/ $4.0 /$ 
recently revealed similar structural arrangements in several clusters of mutually exclusive exons, including the exon 4 and exon 9 clusters in Drosophila Dscam (Yang et al. 2011). All of these structural elements differed from the exon 6 structures in that the docking sites are located in the downstream intron of the cluster. Moreover, a locus control region (LCR) was defined to activate the exon 6 cluster and allow the inclusion of the most proximal exon variant when the docking site is paired with its selector sequence (Wang et al. 2012). However, the intricate regulatory mechanism still remains obscure. For example, a recent deletion analysis of Dscam exon 6 demonstrates a dramatic decrease in the inclusion frequency of most exons 6 , while some exon 6 variants were increasingly included (May et al. 2011), implying that not all of the selections of exon 6 variants may be dependent on the docking site located in the intron downstream from constitutive exon 5. Importantly, further efforts to explore the control mechanism have been hampered by the lack of a sufficient system due to the technical challenges associated with the large size and complexity of the exon cluster.

GATA transcription factors comprise an evolutionarily conserved family of proteins from yeast to mammals that regulate a variety of developmental processes (Patient and McGhee 2002). The Drosophila GATA factor Serpent (Srp) serves diverse and important functions in fat body development, gut differentiation, hematopoiesis, and the humoral immune response (Petersen et al. 1999; Tingvall et al. 2001; Senger et al. 2006; Campbell et al. 2011). Srp encodes different isoforms, generated by mutually exclusive splicing, which contain only a C-finger (SrpC) or both C- and N-fingers (SrpNC) (Waltzer et al. 2002). Mutation analysis indicated that both naturally occurring isoforms could individually rescue blood cell formation in cells lacking an srp null mutation. SrpC and SrpNC isoforms differ in their capacity to activate certain target genes in vivo, and both have common and distinct activities during hematopoiesis and thus extend the range of regulatory options (Waltzer et al. 2002). The lower number of Srp exon variants may facilitate a deeper understanding of the mechanism of mutually exclusive splicing.

In this study, we reveal a molecular model for directing the mutually exclusive splicing of the serpent pre-mRNA, which is based on relative competition between upstream and downstream RNA pairings. Such alternate pairing interactions between the upstream docking site and the downstream selector sequence, or between the downstream docking site and the upstream selector sequence, ensure the inclusion of only one exon variant. Moreover, we demonstrate that bidirectional pairings mediate the splicing outcome of alternative exons in relative pairing strength-correlated mode. Importantly, combined comparative genomics analysis and experimental evidence reveal similar bidirectional structural architectures in exon clusters 4 and 9 of Dscam. Our findings provide a novel mechanistic framework that ensures splicing in a mutually exclusive fashion and further highlight the potentially broad contribution of long-range RNA-RNA interactions to gene regulation.

\section{RESULTS}

\section{Unusual RNA architectures within the srp exon cluster}

Phylogenetic analysis revealed that the $\operatorname{srp}$ gene underwent specific exon duplication in Drosophila (Supplemental Fig. S1) and is subject to mutually exclusive splicing. To decipher the mechanism for $s r p$ mutually exclusive splicing, we first searched for sequences that were conserved in Drosophila. Interestingly, comparative sequence analysis identified four highly conserved intronic elements, one in the intron downstream from exon 3, two between exon 4.1 and exon 4.2, and one upstream of exon 5 (Fig. 1; Supplemental Fig. S2). Surprisingly, these elements are predicted to form two pairs of RNA secondary structures between introns in all of the species surveyed (Fig. 1B; Supplemental Fig. S3). Importantly, the predicted formation of unusual architectures between introns points to a potential mechanism for the mutually exclusive selection of exon 4 variants. As shown in Figure 1B, the inclusion of exon 4.1 would be activated if the latter stem is implemented. However, in the other configuration, where the former stem is implemented, exon 4.2 would be included. Alternatively, both pairs could possibly form a long-range pseudoknot structure. Thus, the presence of these dual docking-site-dependent pairings could constitute a novel mechanistic framework responsible for the mutually exclusive choice of exon variant.

\section{Upstream RNA pairing directs exon 4.2 inclusion}

To confirm this hypothesis, we tested disruptive and compensatory mutations in the dsRNA stem of the Drosophila srp pre-mRNA. We generated a minigene construct containing exons 3-5 and intervening sequences driven by the inducible metallothionein promoter (Fig. 1A). Splice isoforms containing mutually exclusive exons recapitulating the splicing pattern of the endogenous gene could be detected in transfection experiments using Drosophila S2 cells. This system is well suited for delineating important cis-elements involved in the selection of alternative exon 4 . We observed that mutating the upstream docking sequence (Ud) or the downstream selector (Ds), designed to disrupt the upstream pairing interaction, did not cause a significant decrease in the exon 4 inclusion frequency (M1 and M2, Fig. 1C,D). Nevertheless, M1 or M2 mutations resulted in a marked reduction in the exon 4.2 inclusion frequency, while the exon 4.1 inclusion frequency was increased (Fig. 1C,D). Importantly, the reduced efficiency of exon 4.2 inclusion caused by the individual mutations was restored to wild-type levels by compensatory double mutations (M12, Fig. 1C,D). These data indicated that exon 4.2 inclusion was controlled by upstream RNA pairing in D. melanogaster. 
A

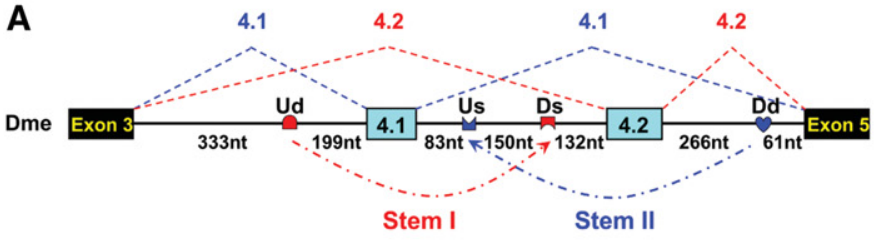

B

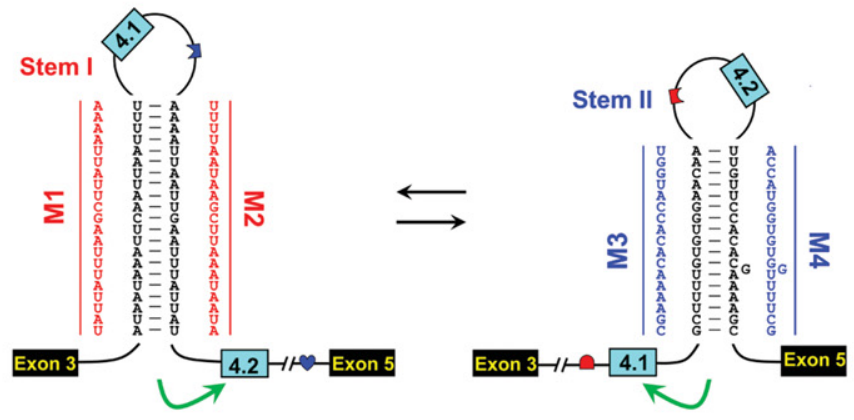

E

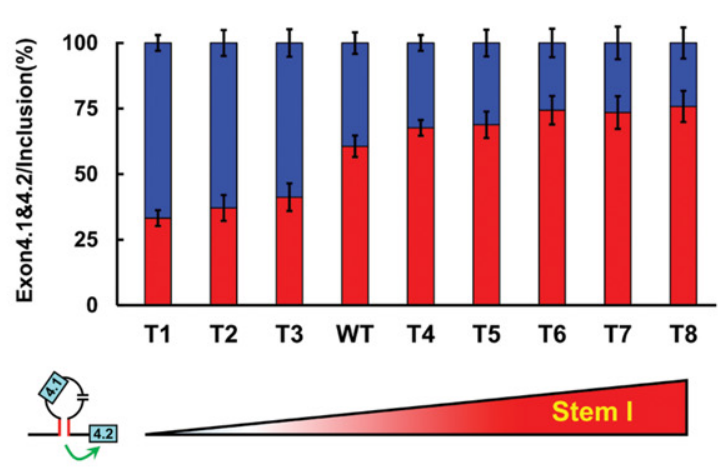

G

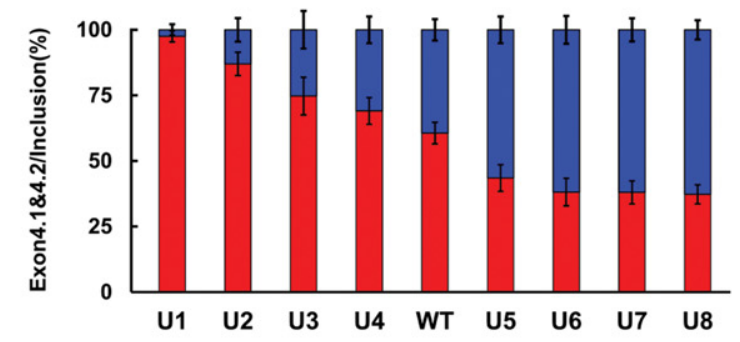

4.1.
C

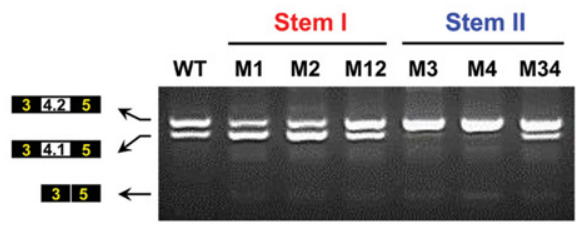

D

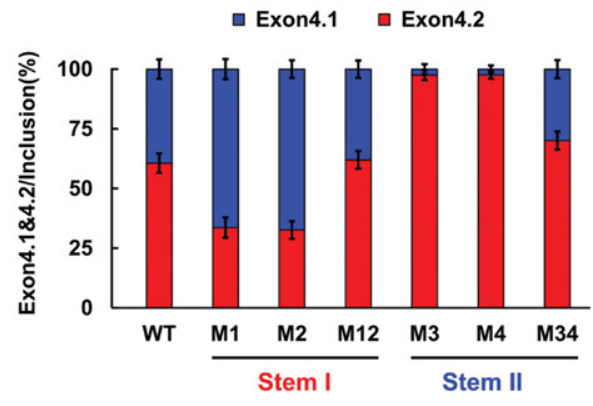

F
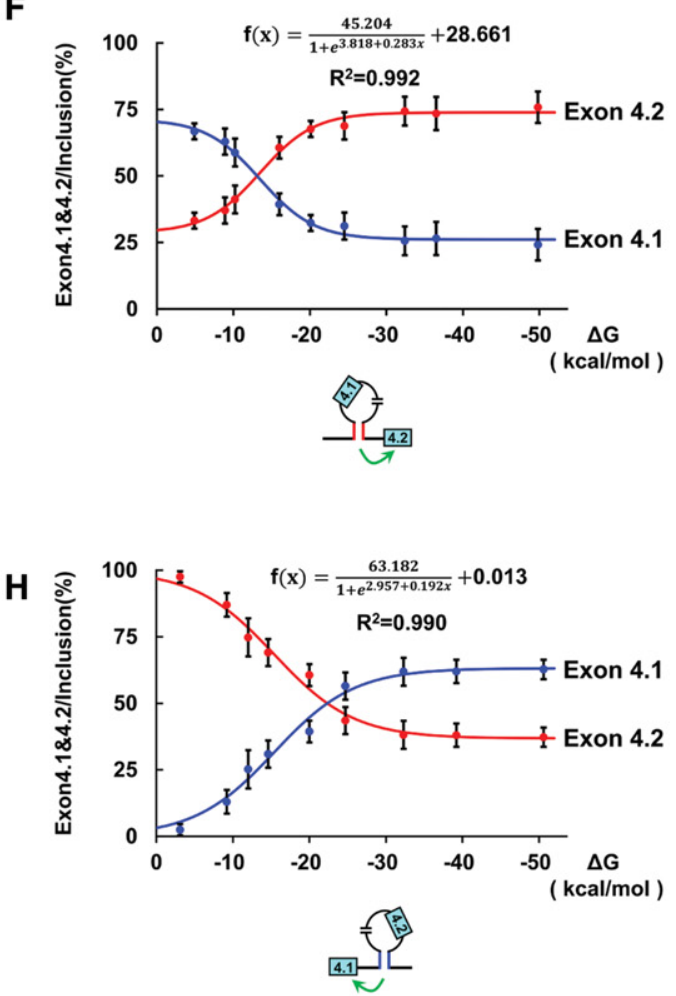

FIGURE 1. Bidirectional RNA pairing controls alternative exon 4 inclusion in Drosophila srp pre-mRNAs. (A) A schematic diagram of the partial premRNA, with constitutive exons depicted as black boxes, mutually exclusive exons as cyan boxes, and introns as lines. The dashed arrow represents the formation of the RNA-RNA interaction. Upstream and downstream docking sites (Ud and Dd; marked by semicircles and hearts, respectively) were complementary to a downstream or upstream selector sequence (Ds and Us; marked by saddle shapes). (B) Predicted RNA pairing of D. melanogaster srp pre-mRNA. A green arrow depicts activation of the inclusion of the proximal exon. Mutations introduced into dsRNA stem are indicated in the left or right mutated sequences (M1-M4). The RNA pairings for other Drosophila species are shown in Supplemental Figure S3. (C) Effects of mutations on exon 4 inclusion are indicated for disruptive single mutations (M1-M4) and compensatory double mutations (M12 and M34). (WT) Wild type. $(D)$ Effects of mutations on exon 4.1 and exon 4.2 selection. $(E)$ The effect of changing the upstream RNA pairing (stem I) strength on exon 4 selection. Predicted RNA pairings for the mutants (T1-T8) are shown in Supplemental Figure S4A. (F) Exon 4.2 inclusion correlated with the upstream RNA pairing (stem I) strength. (G) The effect of changing the downstream RNA pairing (stem II) strength on exon 4 selection. Predicted RNA pairings for the mutants (U1-U8) are shown in Supplemental Figure S4B. $(H)$ Exon 4.1 inclusion correlated with the downstream RNA pairing (stem II) strength. Data are expressed as percentages of the mean $\pm \mathrm{SD}$ from three independent experiments. 
To validate this finding, we generated a series of mutations to assess the contribution of upstream RNA pairing to the selection of exon 4 variants by changing the upstream RNA pairings while leaving the downstream RNA pairings unaffected (Fig. 1E; Supplemental Fig. S4A). When the upstream RNA pairing was weakened by point mutations, the inclusion frequency of exon 4.2 was decreased dramatically. In contrast, strengthening the upstream RNA pairing increased the frequency of exon 4.2 inclusion markedly (Fig. 1E). Analysis of a series of mutations revealed that the exon 4.2 inclusion frequency was strongly correlated with the strength of upstream RNA pairing, whereas the effect of pairing strength on exon 4.2 inclusion was graded (Fig. 1F). Our results indicate that upstream RNA pairing (stem I) specifically directs exon 4.2 inclusion.

\section{Downstream RNA pairing is required for exon 4.1 inclusion}

We used a similar strategy to examine the effect of downstream RNA pairing (stem II) on the selection of alternative exon 4. We found that disruption of the Us or Dd sequences did not result in a remarkable decrease in exon 4 inclusion relative to wild type (M3, M4, Fig. 1C, $\mathrm{D})$; however, the mutations in M3 and M4 nearly abolished exon 4.1 inclusion and exon 4.2 was included exclusively. This result demonstrated that either of the Us and Dd mutations themselves, with a concomitant failure in the downstream RNA pairing interaction (stem II), could specifically abolish exon 4.1 inclusion. Furthermore, combining these two mutations in the same construct (M34) carrying mutations compensatory for the disruptive mutations in stem II of M3 and M4 restored wild-type levels of exon inclusion (Fig. 1C,D).

Consistent with the disruptive and compensatory results, we observed an approximately 1.5 -fold increase in the exon 4.1 inclusion frequency when the downstream RNA pairing was strengthened 1.5-fold (Fig. 1G; Supplemental Fig. S4B). Further strengthening of downstream pairing led to a slight increase in exon 4.1 inclusion, albeit at the low gradient (Fig. 1G). Similar to upstream RNA pairing, we observed a correlation between downstream RNA pairing strength and exon 4.1 inclusion frequency (Fig. 1H; Supplemental Fig. S4B). Collectively, these findings demonstrated that down- stream RNA pairing interaction was critical for exon 4.1 inclusion.

\section{Dual RNA pairings facilitate mutually exclusive splicing fidelity}

The results of our mutational analysis were consistent with a proposed molecular switch model that posits competition between upstream and downstream RNA pairings. To determine how dual RNA pairing could function to ensure the fidelity of exon 4 mutually exclusive splicing, we examined the effect of simultaneous disruption of stems I and II on exon 4 inclusion (Fig. 2A). A small but significant increase in exon 4 skipping was observed when the Ud and Ds sequences were disrupted to abolish stems I and II simultaneously (M1, M2, Fig. 2B,C). Similar results were obtained using other
A

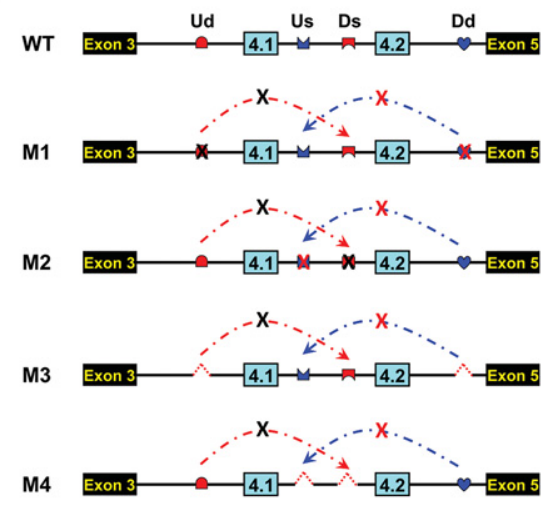

D
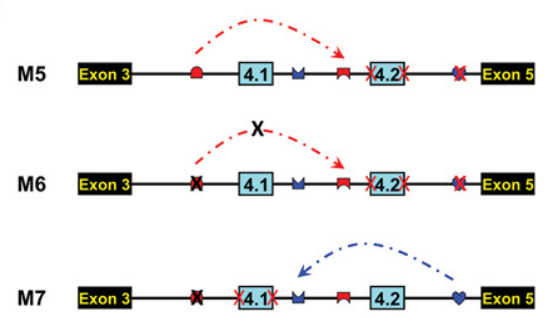

M8

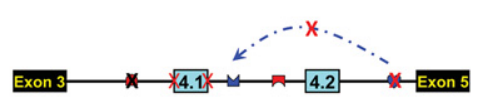

B

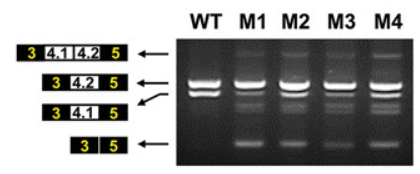

C

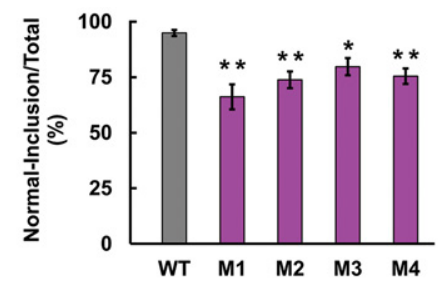

E

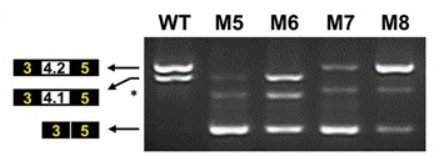

$\mathbf{F}$

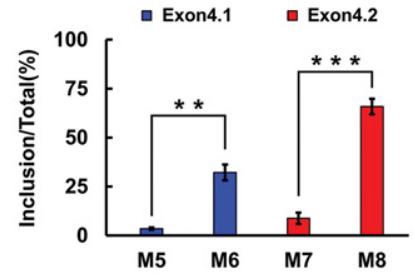

FIGURE 2. Dual RNA pairings facilitate mutually exclusive splicing fidelity. (A) Schematic diagrams of mutant minigene constructs. The symbols used are the same as those in Figure 1. A cross denotes a disrupting mutation, while the dashed arrow plus a cross represents abolishment of the RNA-RNA interaction. $(B)$ The effect of the simultaneous disruption of upstream and downstream pairing on exon 4 inclusion. $(C)$ The simultaneous disruption of upstream and downstream pairing led to increased aberrant splicing of exon 4. (D) Schematic diagrams of the mutants used to evaluate the effect of suppression on inclusion of the in-loop exon by RNA secondary structure. (E) The effect of upstream (or downstream) RNA pairing disruption on exon 4.1 (or exon 4.2) inclusion in the absence of stem II (or stem I). The band marked by “*” is a nonspecific RT-PCR product. $(F)$ The disruption of upstream (or downstream) RNA pairing caused increased exon 4.1 (or exon 4.2) inclusion. Data are expressed as percentages of the mean \pm SD from three independent experiments. $\left(^{*}\right) P<0.05$, (**) $P<0.01$, and (***) $P<0.001$ (Student's $t$-test, two-tailed). 
constructs designed to simultaneously abolish stems I and II (M3, M4, Fig. 2B,C). Such exon 4 skipping, which results in frameshift mutations, cannot be detected in vivo (Supplemental Fig. S5A). Moreover, we observed RT-PCR products containing exon 4.1 and exon 4.2 spliced together as a consequence of the simultaneous disruptive mutations (Fig. 2B). This aberrant splicing is analogous to the inclusion of multiple exon 6 variants in Dscam, which results from the depletion of the binding protein hrp36 (Olson et al. 2007). However, the latter is mechanistically different from the disruption of RNA pairing, which acts by relaxing the suppression of the in-loop exon. Collectively, these results showed that the simultaneous disruption of the upstream and downstream pairing increased the aberrant splicing of exon 4 markedly (Fig. 2C). This strongly suggests that dual RNA pairings may be required for the efficient inclusion of alternative exon 4.

To further examine this process, we generated constructs that contained only active exon 4.1 or exon 4.2 as the internal exon (Fig. 2D). We found that the perturbations of downstream RNA pairing enhanced or even switched the inclusion of exon 4.2, and a similar trend was observed for disruptions of upstream pairing (Fig. 2E,F), consistent with previous observations (Howe and Ares 1997; Yang et al. 2011). These results suggested that dual RNA pairings also function as inherent cis- $^{-}$ acting physical barriers to suppress the in-loop exon to prevent exons 4.1 and 4.2 from skipping and being spliced together.

\section{Dual RNA pairings fine tune exon $\mathbf{4}$ selection}

To delineate why dual pairings are required to regulate mutually exclusive splicing in srp pre-mRNA, we compared the effects of upstream and downstream RNA pairings on exon 4 selection in the presence or absence of competition from the alternate pairing. We assessed the contribution of upstream RNA pairing to the selection of exon 4 variants in $\Delta \mathrm{Dd}$ constructs whose RNAs were unable to form stem II and were therefore not subject to competition from stem II (Fig. 3A). Intriguingly, we observed only a slight change in the exon 4.2 inclusion frequency when stem I was disrupted, in stark contrast to the $\sim 50 \%$ decrease in inclusion frequency in the presence of stem II (Fig. 3A). This trend was reflected by the correlation between the upstream RNA pairing strength and the exon 4.2 inclusion frequency in the presence or absence of competition from stem II (Fig. 3A). In the former case, the 4.2 inclusion frequency ranged from $30 \%$ to $75 \%$ in response to the modulation of stem I strength as evidenced by the steep slope of the curve. In contrast, stem I strength had little effect on the selection of the exon 4 variant in the stem II disruption construct. Thus, our results indicated that upstream RNA pairing lost its regulatory role in alternative exon 4 selection in the absence of competition from downstream pairing.

Interestingly, we observed a different pattern when the effect of stem II strength on exon 4 selection was examined in the presence of stem I or with stem I disrupted (Fig. 3B). We examined the effect of downstream RNA pairings on exon
A

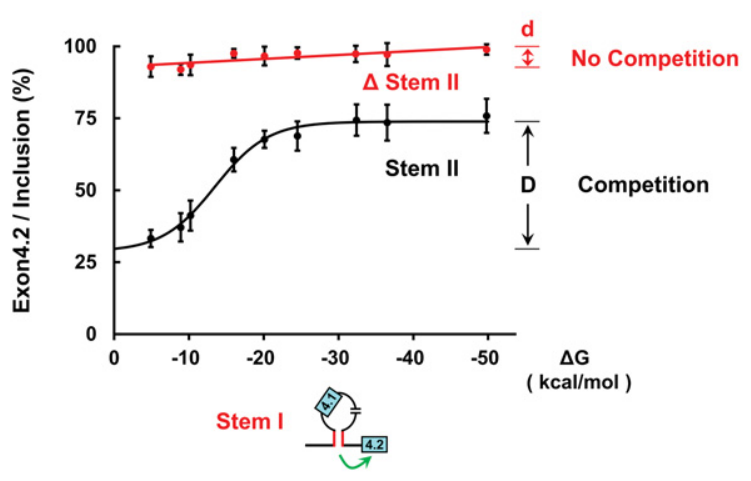

B

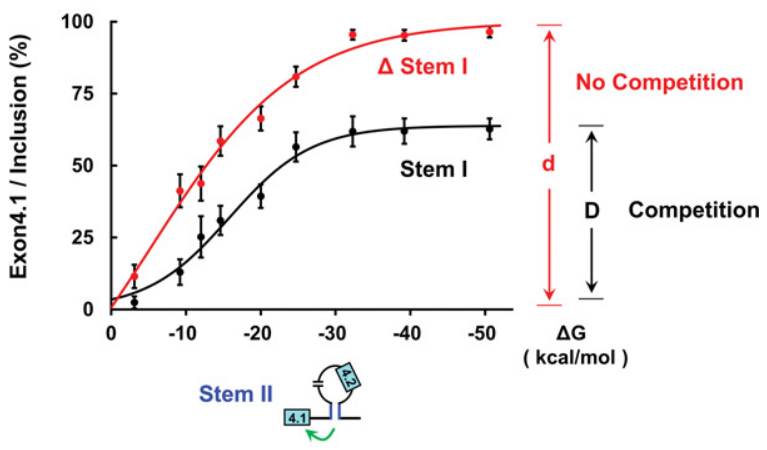

FIGURE 3. Dual RNA pairing may confer a dynamic range to the regulation of alternative splicing. (A) Comparison of the effect of upstream RNA pairing (stem I) on exon 4.2 inclusion in the presence versus absence of competition from stem II. "D" and " $d$ " denote tunable breadth in the presence versus absence of competition from stem II. (B) Comparison of the effect of downstream RNA pairing (stem II) on exon 4.1 inclusion in the presence versus absence of competition from stem I. Data are expressed as percentages of the mean \pm SD from three independent experiments.

4.1 selection in $\Delta \mathrm{Ud}$ constructs which could not form stem I. As expected, weakening the upstream RNA pairing decreased the exon 4.1 inclusion frequency dramatically, whereas strengthening the upstream pairing had the opposite effect (Fig. 3B). However, the change was much greater than that in the presence of stem II (Fig. 3B), suggesting that the stem II pairing acted as a rheostat. Collectively, these results imply that dual RNA pairing may confer a dynamic range to the regulation of alternative splicing.

\section{Exon 4 selection correlates with competition between upstream and downstream pairings}

Our results above showed that upstream or downstream RNA pairings regulate the selection of alternative exon 4 in a strength-correlated manner (Fig. 1F,H). To elucidate how these dual RNA pairings act in concert to modulate exon selection, we generated a series of mutations to alter the competition between upstream and downstream RNA pairings to assess their contributions to the selection of exon 4 variants. These mutations included combinations of changes in the upstream and downstream pairing strengths. We found that 
strengthening stem I reversed the effect of strengthening stem II on exon 4.1 inclusion and vice versa. In particular, simultaneous strengthening or weakening of stems I and II to similar degrees did not have a remarkable effect on exon 4 selection (Fig. 4A,B). Exon 4.2 inclusion frequency was more strongly correlated with the ratio of upstream to downstream RNA pairing strengths than with the absolute strengths of the upstream or downstream RNA pairings (Supplemental Fig. S6). Therefore, we concluded that alternative exon 4 selection was determined by the relative competitiveness between upstream and downstream RNA pairings.

This view was further supported by the ratio analysis of the selection of alternative exon 4 in the endogenous srp gene of D. melanogaster. In this study, the inclusion of exon 4.2 was generally predominant in all tissues examined although the predicted free energy of the upstream secondary structure was slightly weaker than that of the downstream secondary structure (Supplemental Fig. S5A-C). Even if we mutated the pairing strength of stem II to be identical to that of stem I, the exon 4.2 inclusion frequency was much higher than that of exon 4.1 (Supplemental Fig. S5D,E), suggesting that the inclusion frequency of the alternative exon cannot be explained merely by the strength of certain pairings. In addition, we observed that the intronic distances between the $5^{\prime}$ splice site and the upstream selector were shorter than the distances between the upstream selector and the $3^{\prime}$ splice site in Drosophila (Supplemental Fig. S5F), which cannot account for differences in exon 4.1 and 4.2 inclusion frequencies. Remarkably, the $5^{\prime}$ and $3^{\prime}$ splice sites of exon 4.2 were much stronger than those of exon 4.1 across Drosophila species (Fig. 5A), consistent with the more frequent inclusion of exon 4.2. This observation leads us to speculate that the splice site strengths of exon 4 variants likely contribute to their different inclusion frequencies.

\section{Splice site strength modulates RNA-pairing-mediated alternative exon 4 selection}

To further elucidate how splice site strength contributes to the pairing-mediated selection of alternative exon 4 , we per-
A

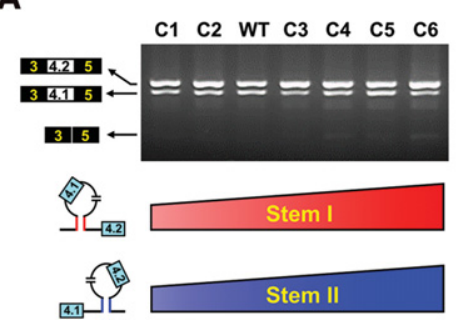

B

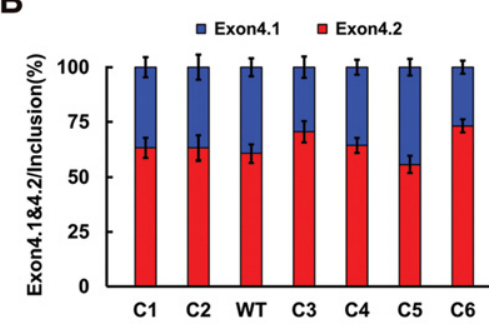

FIGURE 4. Regulation of mutually exclusive splicing in relative pairing strength-dependent mode. (A) The effect on exon 4 inclusion by simultaneously changing the upstream and downstream pairing strength. C1-C6 denotes the combined mutations on dual pairings (Supplemental Fig. S4C). (B) Quantization of the data in $A$. Data are expressed as percentages of the mean \pm SD from three independent experiments. formed a series of experiments in which the $5^{\prime}$ or $3^{\prime}$ splice site of alternative exon 4.1 was strengthened (Fig. 5B). We observed that exon 4.1 was included exclusively when the $3^{\prime}$ splice site of exon 4.1 was strengthened relative to that of exon 4.2 (Fig. 5C,D). Conversely, the same trend was seen for "weak to strong" 5 ' splice site mutations. These results support the idea that the weak splice site of alternative exon 4.1 is responsible, at least in large part, for the lower frequency of exon 4.1 inclusion. This was reinforced by altered splice site strength in combination with disruption of the RNA pairing interaction. When a single $5^{\prime}$ or $3^{\prime}$ splice site of exon 4.1 was optimized, exon 4.1 was partially or largely included even in the absence of downstream RNA pairing (Fig. $5 \mathrm{C}, \mathrm{D})$. These data indicated that splicing of the mutant minigene was partially RNA pairing-independent, implying that downstream RNA pairing activated alternative exon selection by promoting the recognition of the weak splice site.

In contrast to exon 4.1, exon 4.2 contained comparatively stronger $5^{\prime}$ and $3^{\prime}$ splice sites evidenced by the fact that exon 4.2 was included even in the absence of upstream RNA pairing (Fig. 5E,F), suggesting that exon 4.2 inclusion was partially RNA pairing-independent. To determine to what extent RNA pairing contributed to alternative exon 4.2 inclusion, we created splice site mutations either alone or in combination with pairing disruption. As expected, we found that weakening the $5^{\prime}$ or $3^{\prime}$ splice site decreased exon 4.2 inclusion substantially, whereas strengthening the $5^{\prime}$ or $3^{\prime}$ splice site had the opposite effect (Fig. 5E,F). The same trend was seen in the absence of upstream RNA pairing (Fig. 5E,F). Notably, exon 4.2 was included rarely in the absence of upstream RNA pairing when the $5^{\prime}$ splice site was weakened. These results implied that competition between the splice sites of alternative exons was involved in the pairing-mediated selection of alternative exon 4 and that the splice site and RNA pairing strengths compensated for each other. When the pairing strengths were similar, the stronger splice site of alternative exon 4.2 allowed for faster recognition and splicing and more frequent inclusion than alternative exon 4.1 in the mature mRNA. These results at least partially explain the differences in exon 4.1 and 4.2 inclusion frequencies and strongly suggest multiple ways to modulate the inclusion of alternative exons based on a subtle noncoding mutation.

\section{Both RNA pairings display distinct approximation effects}

We sought to determine how two nonsequence-specific RNA pairing guides specifically activated the selection of alternative exon 4. Two general scenarios, which may not be mutually exclusive, could provide clues to the nature of the mechanism. In the first scenario, the RNA structures must be bound by and 
A

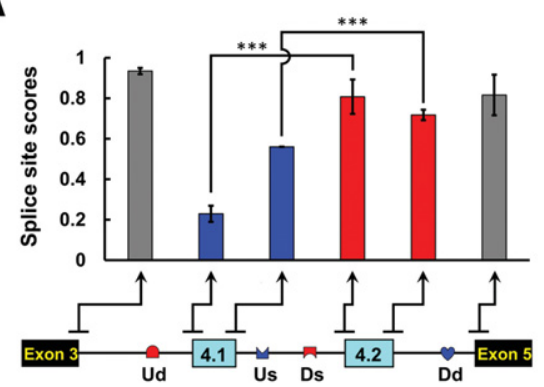

C

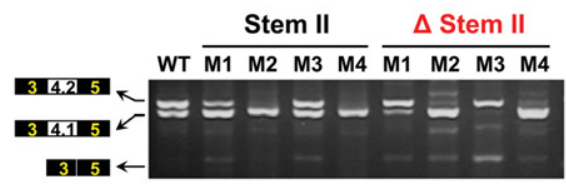

D

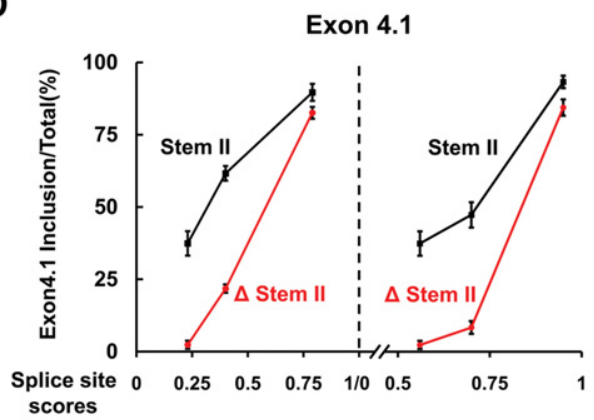

B

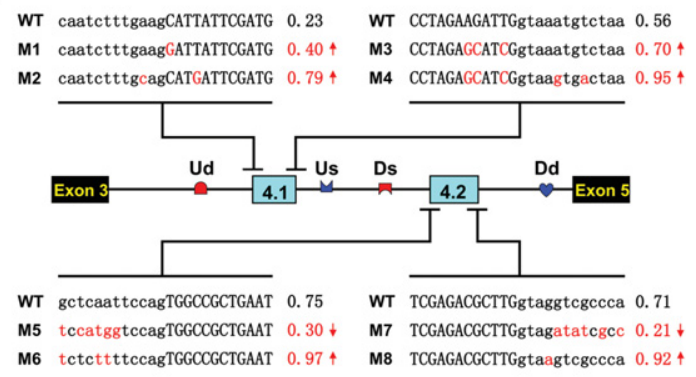

E

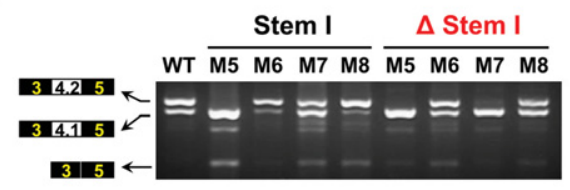

$\mathbf{F}$

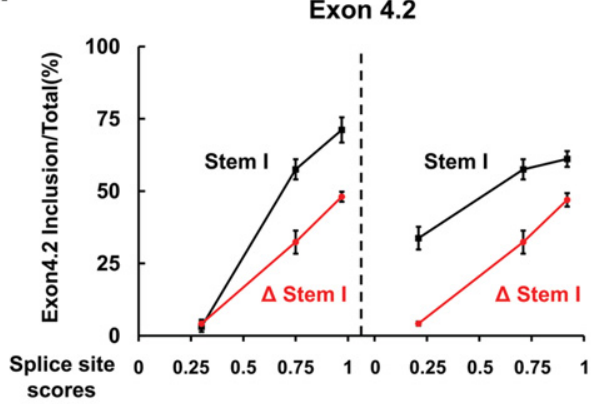

FIGURE 5. Splice site strength modulates RNA pairing-mediated alternative exon 4 selection. (A) Splice sites built on exon clusters in Drosophila srp genes. The $5^{\prime}$ and $3^{\prime}$ splice site motif scores were calculated using the splice site predictor (Reese et al. 1997) (range is 0-1, with higher values predicting stronger splice sites). Statistical analysis revealed that the splice site of exon 4.2 is significantly stronger than that of exon 4.1 . $\left({ }^{* * *}\right) P<0.001$ (Student's $t$-test, two-tailed). Symbols used are the same as those in Figure 1. (B) Splice site sequences of the wild-type and mutants, with the scores on the right. Uppercase letters indicate exon sequences; lowercase letters indicate intron sequences. Mutated nucleotides are marked in red. $(C)$ Effect of strengthening the weak splice sites on alternative exon 4.1 inclusion. (D) Comparison of the effect of strengthening the weak splice sites on alternative exon 4.1 inclusion in the presence versus disruption of downstream RNA pairing. (E) Effect of changing the splice sites on alternative exon 4.2 inclusion. $(F)$ Comparison of the effect of changing the splice sites on alternative exon 4.2 inclusion in the presence versus disruption of upstream RNA pairing. Data are expressed as percentages of the mean \pm SD from three independent experiments.

interact with regulatory proteins without sequence specificity to mediate the inclusion of alternative exon 4. Alternatively, RNA structures could act as distant upstream and downstream elements via base-pairing as was shown previously (Muh et al. 2002; Baraniak et al. 2003; Yang et al. 2011). To investigate these two possibilities, we created a series of constructs with deletions of Us and Dd, and/or the loop sequence (Fig. 6A). These deletions were designed to mimic the approximation of sequences caused by the inter-intronic Us-to-Dd pairing (stem II). RT-PCR analysis revealed that exon 4.1 was predominantly included ( 90\%) (M3, Fig. $6 \mathrm{~B}, \mathrm{C})$, while the perturbation of downstream pairing caused a remarkable decrease in exon 4.1 inclusion (M4) and enhanced exon 4 skipping. Importantly, the exon 4.1 inclusion frequency was not affected significantly even though the construct lacked the Us-to-Dd RNA stem (M6, Fig. 6B,C). This change in splicing pattern was consistent with previous studies (Muh et al. 2002; Baraniak et al. 2003; Yang et al. 2011) and provides strong evidence that exon 4.1 inclusion is dependent on the formation of downstream pairing to bring splice sites (or/and the elements) in proximity, but the structure itself is not required.

Interestingly, a distinct pattern was observed when we examined the approximation effect of Ud-to-Ds RNA pairing (stem I) on the selection of alternative exon 4.2 (Fig. 6D-F). We observed that exon 4.2 was frequently included $(\sim 70 \%)$ in the presence of the upstream pairing (M9, Fig. 6E,F), as in the construct that lacked $\mathrm{Ud}$, Ds and intervening sequence (M12). However, exon 4.2 inclusion was slightly but not significantly reduced by perturbation of the upstream pairing (M10). These observations demonstrate that RNA pairingfacilitated approximation may not be evident. These results are supported by the fact that alternative exon 4.2 was included frequently even after the simultaneous disruption of stems I and II (Fig. 2A,B). This approximation pattern discrepancy was compatible with the much stronger splice 
A

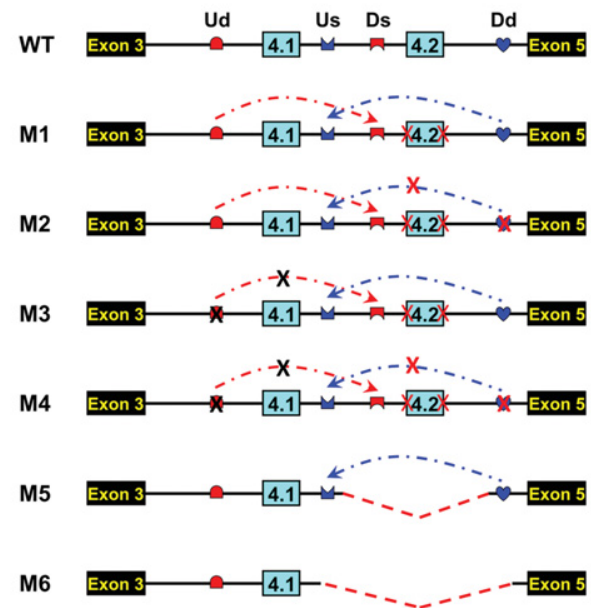

D

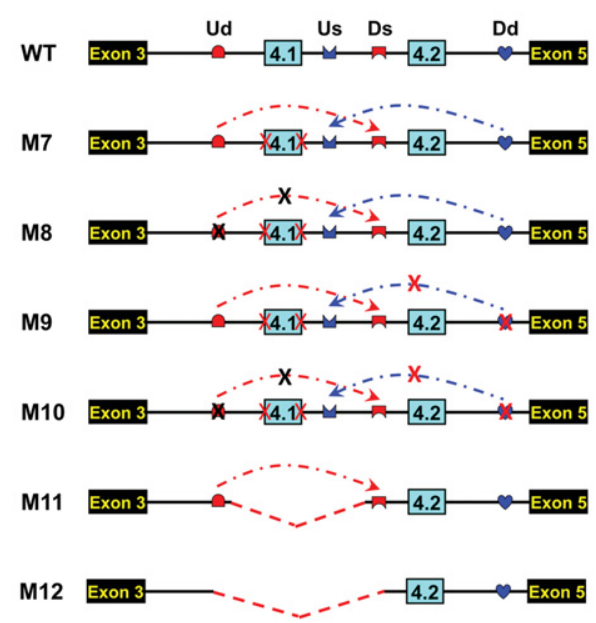

B

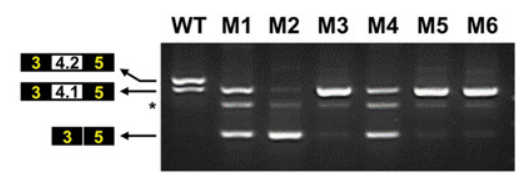

C

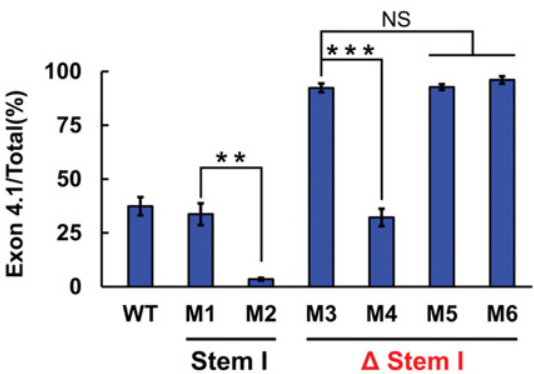

E

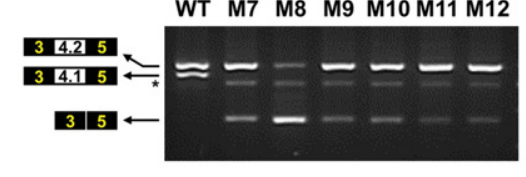

$\mathbf{F}$

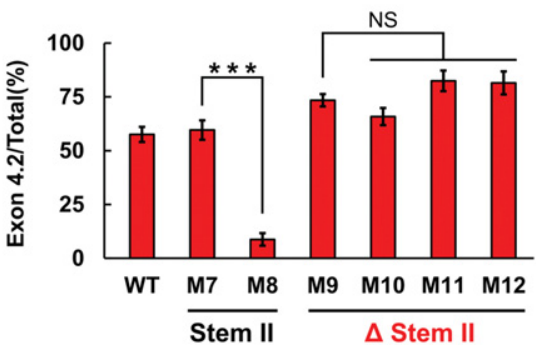

FIGURE 6. Different approximation pattern of bidirectional pairings. (A) Overview of the minigene constructs used to mimic the approximation of sequences caused by the Us-to-Dd pairing (stem II). A cross in Us or Dd denotes the disrupting mutation. Symbols used are the same as those in Figure 1. (B) Effects of exon 4.1 selection by a series of deletions and mutations. The band marked by “*” is a nonspecific RT-PCR product. (C) Quantization of the data in $B$. Data are expressed as percentages of the mean \pm SD from three independent experiments. $\left(^{* *}\right) P<0.01,\left({ }^{* * *}\right) P<0.001$ (Student's $t$-test, two-tailed). These data indicate that RNA pairing interactions function to approximate upstream and downstream sequences. $(D)$ Overview of the minigene constructs used to evaluate the importance of approximating sequences caused by Ud-to-Ds pairing (stem I). (E) Effect of a series of deletions and mutations on exon 4.2 inclusion. $(F)$ Quantization of the data in $E$.

site strength of alternative exon 4.2 than exon 4.1. These results indicated that upstream pairing acted to enhance the exon 4.2 splicing rate and efficiency by bringing splice sites in proximity, thereby resulting in striking preferences in competition with downstream-pairing-dependent exon 4.1 splicing.

\section{Mimicking the bidirectional expansion of dual pairing}

To investigate whether the dual pairing could support mutually exclusive splicing of three or more exon clusters, we mimicked exon expansion by duplicating either exon 4.1 or exon 4.2 or by combining them. We inserted exon 4.1 and its flanking sequence into the exon 4 cluster and evaluated the splicing pattern and efficiency of the resulting construct
(Fig. 7A). In this case, an upstream selector sequence required to ensure mutually exclusive splicing was duplicated simultaneously with the exon 4.1 expansion. Little aberrant splicing, including exon skipping, was detected (Fig. 7B). Exon-specific restriction digestion revealed that three exon variants, including nascent exon $4.1^{*}$, were alternatively spliced in the mutually exclusive manner (In1, Fig. 7B,C). This result demonstrated that mutually exclusive splicing was not disrupted by expansion of the two-exon cluster in srp to a three-exon cluster. In addition, a similar splicing pattern was observed with an insertion construct containing a duplication of exon 4.2 and its flanking sequence (In2, Fig. $7 \mathrm{~B}, \mathrm{C})$. Furthermore, mutually exclusive splicing was not disrupted within the cluster after the three-exon cluster was expanded to a four-exon cluster (In3, Fig. 7B,C). The elegant 


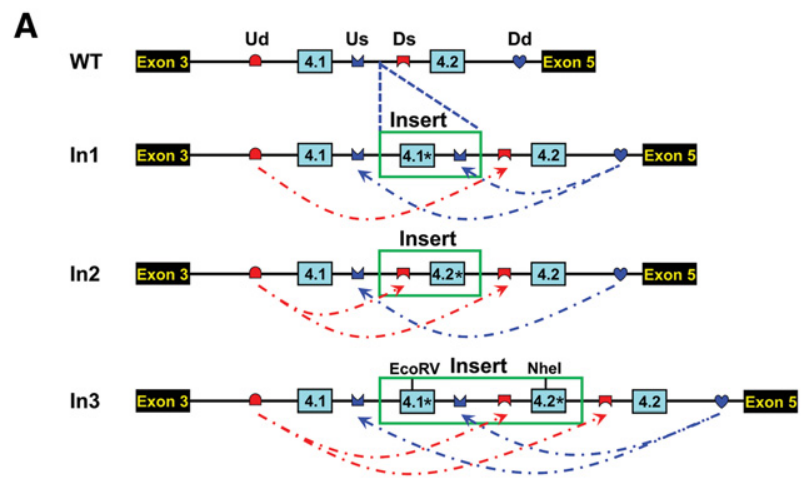

B

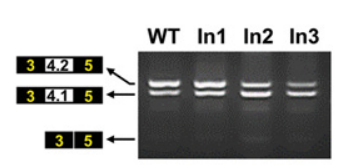

C

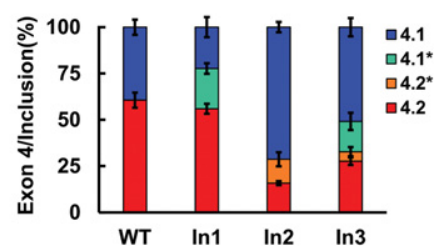

FIGURE 7. Mimicking the expansion of bidirectional pairings. $(A)$ Schematic diagrams of wild-type (WT) and mutant minigene constructs to mimic expansion of the bidirectional pairing system. Mutation was performed to produce a specific enzyme site to discriminate the original exon 4.1 or 4.2 (exon $4.1^{*}$ or $4.2^{*}$ ). (B) RT-PCR products from RNA isolated from Drosophila S2 cells transiently transfected with the insertion constructs. $(C)$ The inclusion analysis of alternative exon 4 . Quantization of the frequency of exon 4 utilization was determined by variant-specific restriction digestion. Data are expressed as percentages of the mean \pm SD from three independent experiments.

arrangement of intronic elements facilitates the heritable expansion of the control system in accompaniment with bidirectional expansion of exon clusters thus preserving the integrity of mutually exclusive splicing in an ever-increasing exon cluster. This suggests that the dual pairing system faces little regulatory conflict during the evolutionary expansion of an exon cluster.

\section{Dscam exon clusters revisited}

Whether this intriguing mechanistic framework operates in other genes remains to be seen. Previous studies indicated that single selection of variant exons is directed by RNA pairing interaction between a unique docking site and a selector sequence in the exon 4, 6, and 9 clusters in Drosophila Dscam (Graveley 2005; Anastassiou et al. 2006; Yang et al. 2011). However, this model was based on relatively small-scale data from limited Drosophila species. Therefore, we revisited Dscam exon clusters 4, 6, and 9 using a larger number of different arthropod species for higher resolution. Consistent with our previous study (Yang et al. 2011), we found no convincing evidence for the arrangement of dual docking sites and secondary structural interactions within the exon 4 cluster in Drosophila species, nevertheless we discovered conserved dual docking sites within Dscam exon 4 clusters in hymenopteran species (Fig. 8A). In these cases, a conserved intronic element downstream from constitutive exon 3 was complementary to an intronic element within alternative exon 4 in 11 hymenopteran species, while a conserved intronic element upstream of constitutive exon 5 was complementary to an upstream intronic element (Supplemental Fig. S7). Given the large size of the hymenopteran Dscam exon 4 cluster and the concomitant technical difficulties, we sought to generate deletion constructs to test the effects of RNA pairing on splicing in silkworm cells (Fig. 8A,B). Since exon 4.4 was seldom included in the minigene constructs using $\mathrm{BmN}$ cells, we did not demonstrate the proposed dsRNA stem in exon 4.4 inclusion. However, mutating the upstream docking site (M1) or the downstream selector (M2) significantly increased exon 4.1 inclusion (Fig. 8C-E). Importantly, compensatory double mutations (M12) reverted the efficiency of exon 4.1 inclusion to wild-type level. Moreover, stronger pairing in compensatory double dsRNA (M34) enhanced such inhibitory effects on exon 4.1 inclusion (Fig. 8D-F). These observations were consistent with the notion that the RNA secondary structure could enhance in-loop exon skipping (Howe and Ares 1997), thus strongly verifying that these elements act to regulate exon 4 inclusion through formation of upstream RNA secondary structures.

We attempted to clarify the effect of the downstream dsRNA (stem II) on Dscam exon 4.1 inclusion. Disruption of this downstream structure by individual mutations (M5, M6) led to a dramatic decrease in exon 4.1 inclusion; expectedly, a structure-restoring double mutation (M56) restored the efficiency of exon 4.1 inclusion to wild-type level (Fig. 8D,G). Moreover, compensatory double mutation (M78) to strengthen pairing increased the efficiency of exon 4.1 inclusion up to $\sim 80 \%$, much higher than the wild-type level (Fig. $8 \mathrm{D}, \mathrm{G})$. Analysis of the series of mutations indicates that downstream pairing interaction acts in strength-dependent mode (Fig. 8H). Importantly, some evidence of compensatory structural evolution exists within core regions in downstream dsRNAs (Supplemental Fig. S7D). These data validate that the inclusion of some exon 4 variants is dependent on base pair interactions between the downstream docking site and upstream selector sequence. Taken together, these results demonstrate that bidirectional RNA pairings control Dscam exon 4 inclusion in A. mellifera.

We have not found strong evidence for dual docking sites within exon 6 clusters in the insect species investigated. Interestingly, we discovered dual docking sites and their guided structural architecture within Dscam exon 9 clusters in lepidopteran species (Fig. 9A; Supplemental Fig. S8). To validate the bidirectional RNA pairing interaction, we tested the effect of the silkworm dsRNA on exon 9 inclusion using disrupting and compensatory mutations (Fig. 9B). Disrupting mutations (M1, M2) on upstream stem I led to a dramatic decrease in silkworm exon 9.8 (Fig. 9C-E). The reduced efficiency of silkworm exon 9.8 inclusion caused by individual mutations (M1, M2) was restored by compensatory double mutations (M12) (Fig. 9C-E). Likewise, disruption of this downstream 
A

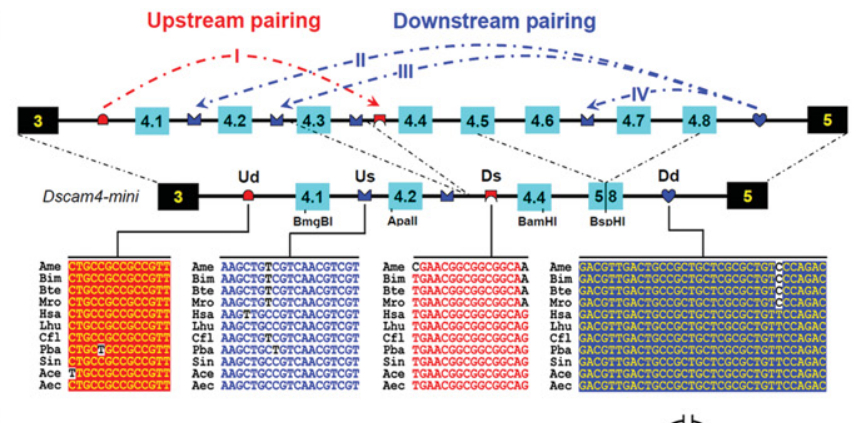

B

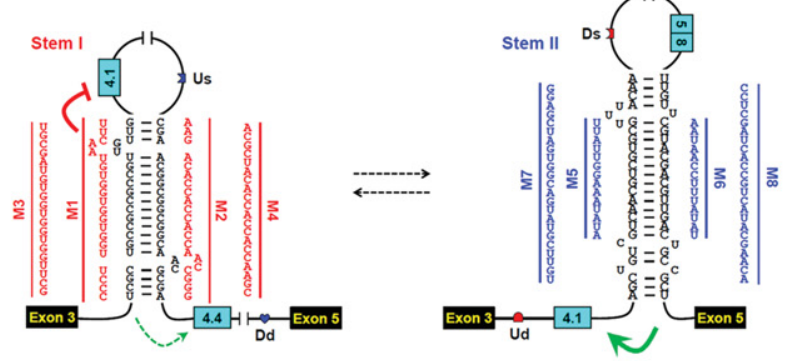

C

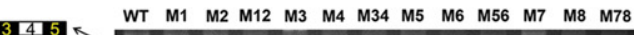

D

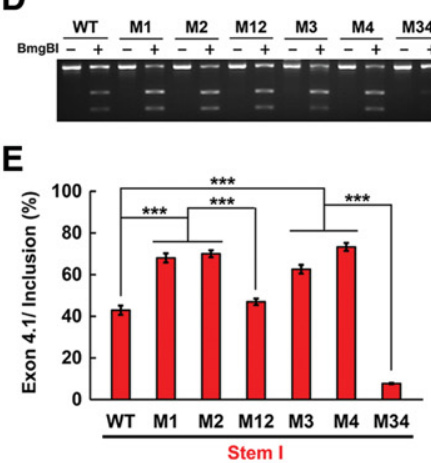

$\mathbf{F}$

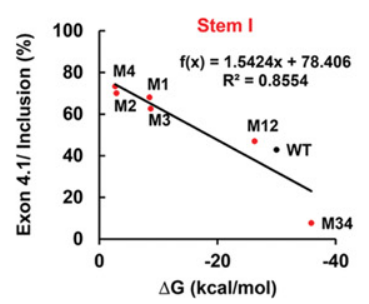

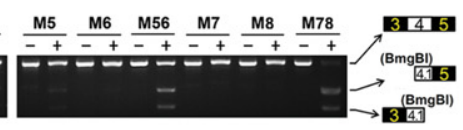

G

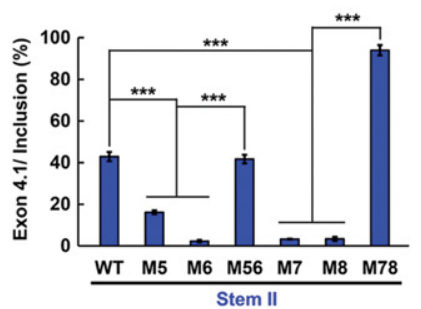

H

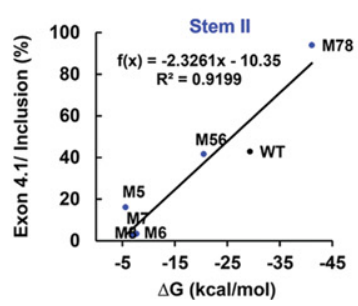

FIGURE 8. Similar bidirectional pairings were experimentally confirmed in exon cluster 4 of hymenopteran Dscam. (A) Overview of the deleted minigene constructs of Apis mellifera. Symbols used are the same as those in Figure 1. The nucleotides with greatest identity to the dual docking sites and selector sequences are shown in different colors. $(B)$ The predicted RNA pairings of Dscam pre-mRNA. Mutations introduced into dsRNA are indicated on the left or right mutated sequences (M1-M8). The green arrow depicts activation of the inclusion of the proximal exon, while the dashed green arrow depicts the potential activation. $(C)$ Effects of bidirectional RNA pairings on exon 4 inclusion are indicated for disruptive single mutations (M1-M8) and compensatory double mutations (M12; M34; M56; M78). The band marked by “*” is a nonspecific RTPCR product. $(D)$ Effects of RNA pairings on exon 4.1 inclusion. The PCR lanes represent the single exon 4-containing band cut from C. Exon 4.1-specific restriction digestion was performed to analyze the frequency of exon 4.1 utilization. $(E)$ Quantization of the data in left panel of $D$. Data are expressed as percentages of the mean $\pm S D$ from three independent experiments. $\left.{ }^{* * *}\right) P<0.001$ (Student's $t$-test, two-tailed). ( $F$ ) Exon 4.1 inclusion negatively correlated with the strength of upstream RNA pairing. $(G)$ Quantization of the data in the right panel of $D$. $(H)$ Exon 4.1 inclusion positively correlated with the strength of downstream RNA pairing.

structure (stem III) by individual mutations (M3, M4) eliminated silkworm exon 9.5 inclusion, but its reduced efficiency was restored by compensatory double mutations (M34) (Fig. 9C-E). Moreover, the reduced efficiency of silkworm exon 9.6 inclusion in other deleted minigenes caused by individual mutation of the downstream stem (stem IV) was largely restored by compensatory double mutations (M54) (Fig. 9C-E). Because the targeted exons for dsRNA II and V were seldom included in transfection experiments with silkworm cells, we have not yet experimentally demonstrated their proposed structures. However, they are strongly supported by their evolutionary conservation and some compensatory mutations in Danaus plexippus (Supplemental Fig. S8B). Moreover, this architecture is evolutionarily conserved in hymenopteran species, although the primary sequence is highly diverged (Supplemental Fig. S9). Moreover, clear evidence of compensatory structural evolution and evolutionary intermediates exists within dsRNA regions in hymenopteran (Supplemental Fig. S9). Collectively, these data demonstrate that the bidirectional RNA pairings function in the inclusion of Dscam exon 9 in lepidopteran and hymenopteran species.

\section{DISCUSSION}

This study explores the role of the unprecedented architecture of RNA-RNA interactions in mutually exclusive splicing. In silico approaches such as comparative genomics and structural prediction are useful for identifying base-pairing interactions (Plocik and Graveley 2013); however, highly complex RNA interactions may be extremely difficult to predict (Cruz and Westhof 2009). The RNA bidirectional structural architectures identified in our study were not detected using recent high-throughput RNA structure sequencing techniques (Daines et al. 2011; Li et al. 2012), nor were they discovered by in silico approaches, although stem II of the Drosophila srp pre-mRNA was predicted (Raker et al. 2009; Pervouchine 2014). Moreover, most RNA structural studies 
A

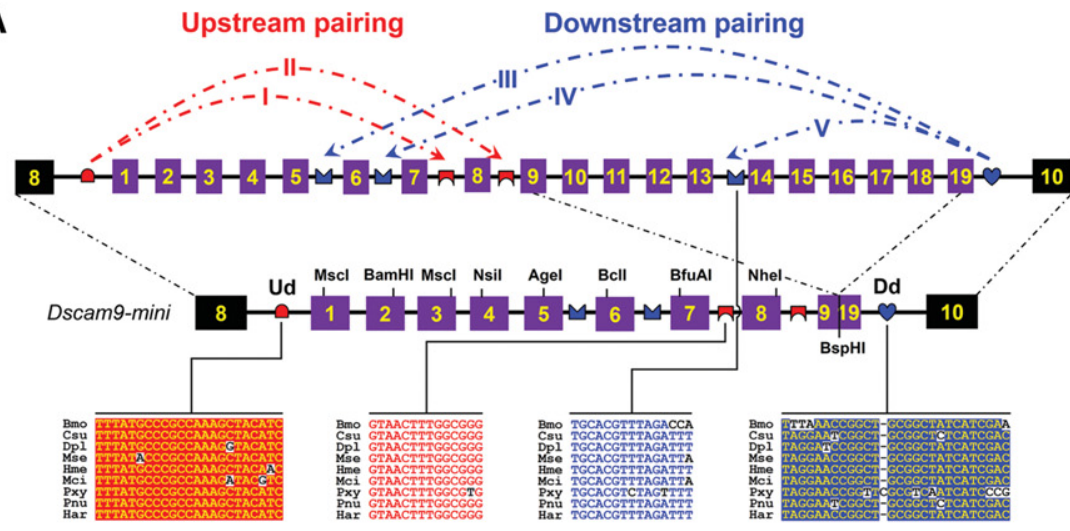

B

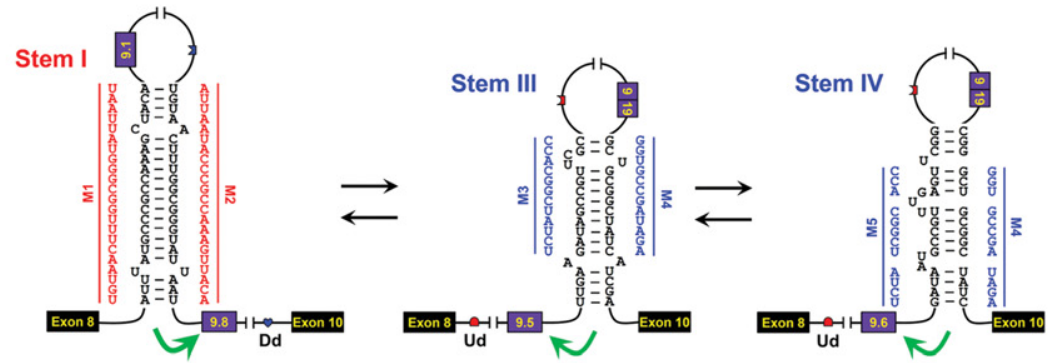

C

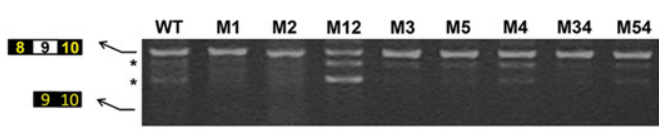

D

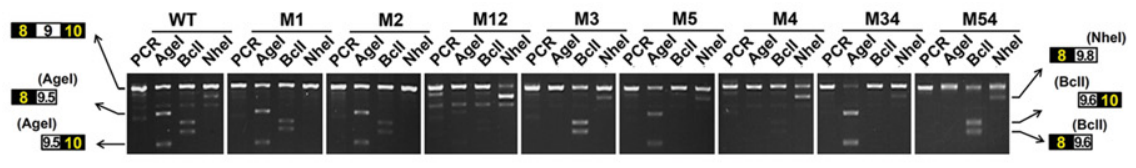

E

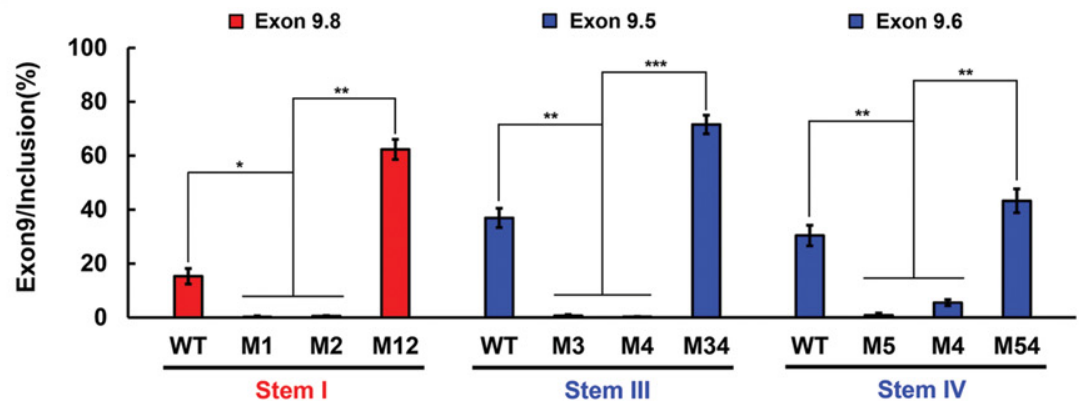

FIGURE 9. The evolutionarily conserved bidirectional pairings within Dscam exon 9. (A) Overview of the silkworm minigene constructs. Symbols used are the same as those in Figure 1. The nucleotides with greatest identity to the docking sites and selector sequences are shown in different colors. (B) Schematic diagrams of the minigene constructs for the predicted RNA pairings. Mutations introduced into dsRNA are indicated on the left or right mutated sequences (M1-M5). (WT) Wild type. (C) Effects of bidirectional intronic RNA pairings on exon 9 inclusions for disruptive single mutations (M1-M5) and compensatory double mutations (M12; M34; M54). The band marked by “*” is a nonspecific RT-PCR product. (D) Effects of bidirectional RNA pairings on exon 9 selections. The PCR lanes represent the single exon 9-containing band cut from $C$. Variant-specific restriction digestion was performed to analyze the frequency of exon 9 utilization. (E) Quantization of the data in $D$. Data are expressed as percentages of the mean \pm SD from three independent experiments. $\left({ }^{*}\right) P<0.05,\left({ }^{*}\right) P<0.01,\left({ }^{* * *}\right)$ $P<0.001$ (Student's $t$-test, two-tailed). focus on the role of a local RNA duplex in alternative splicing and other processing. More than five years after the discovery of competing pairing based on an upstream docking site in the Dscam exon cluster 6 (Graveley 2005), we explored the competing pairing in the $14-3-3 \xi$ gene and in Dscam exon clusters 4 and 9 based on a downstream docking site (Yang et al. 2011). Ten years after the initial model was described (Graveley 2005), this study further revealed that the upstream and downstream docking sites and their base-pairing are present in the same exon cluster. Combining informatics and evolutionary analysis with experimental data, we propose a bidirectional pairing model to explain the splicing mechanism of commitment to a single choice from a tandem array of exons (Fig. 10).

Obviously, dual docking sites heavily complicate pairing-guided mutually exclusive selections of exon variants. In contrast with absolute mutually exclusive pairing in the previous unidirectional pairing model (Graveley 2005; Yang et al. 2011), it is possible that both pairs are not mutually exclusive, but occur simultaneously through the formation of a long-range pseudoknot (i.e., srp) or double hairpin (i.e., Dscam exon 9 cluster). However, the former is contradictory in regulation; for example, upstream RNA pairing activated exon 4.2 inclusion, while downstream RNA pairing simultaneously suppressed it. Moreover, long-range tertiary pseudoknot structures are frequently essential for function. For example, telomerase relies on a core pseudoknot within its RNA component to synthesize telomeric DNA (Blackburn and Collins 2011). Similarly, the formation of a pseudoknot is required for efficient coronavirus ribosomal frameshifting during protein synthesis (Brierley et al. 1989). In contrast, no precedent exists for a requirement for a long-range pseudoknot structure governing specific exon inclusion in a mutually exclusive mode. Our results indicate that inclusion of a single exon 4 is similar to the WT in the disruption of alternate pairing (Fig. 1C). Furthermore, simultaneous strengthening or weakening of both pairs to similar degrees had no 
A
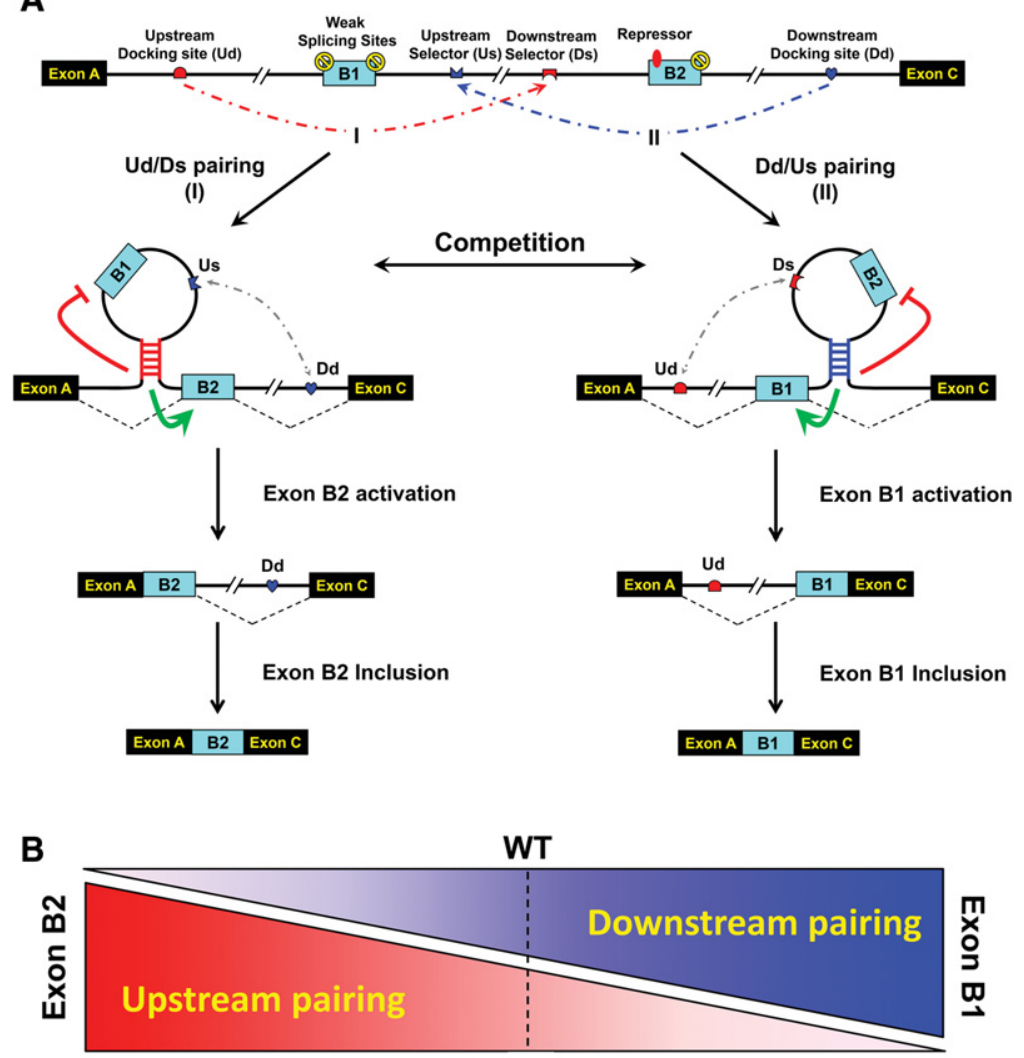

FIGURE 10. Bidirectional RNA pairing model for coordinating mutually exclusive splicing. $(A)$ Bidirectional pairing model. Symbols used are the same as those in Figure 1. The dashed gray arrow is depicted as the potential pairing interaction. For an alternative exon $\mathrm{B}$ to be included in the mature mRNA, either of the docking sites alternately interacts with their selector sequence. For example, when the Us pairs with the Dd, the RNA pairing interaction functions to promote the recognition of the weak splice site, thereby activating the splicing of exon B1 to constitutive exon C. In this case, only the exon B1 variant is included in the mRNA, because the remaining exon B2 might be actively repressed by downstream pairing in combination with other players such as weak or suboptimal splice sites (yellow circle) and splicing repressors (red oval). While the Ud pairs with the Ds, such RNA pairing interactions serve to activate or enhance the inclusion of alternative exon B2. Alternatively, both pairs occur simultaneously through the formation of a longrange pseudoknot. In this case, the more quickly spliced exon would be included in the mRNA, while the other is spliced out. (B) Upstream pairing (red) and downstream pairing (blue) are competitive but regulate the inclusion of the alternative exon.

remarkable effect on exon 4 selection (Fig. 4A,B). Therefore, we propose that these elements would not tend to act via the formation of a pseudoknot, but via competing RNA-RNA pairing interactions in vivo. Alternatively, even if both pairs occur simultaneously, the mutually exclusive inclusion of alternative exon 4 may depend on the kinetics of pairing-based splicing. In this case, the more quickly spliced exon would be included in the mRNA while the other is spliced out (Fig. 10A). Thus, in any specific structure, only one exon variant would be included in each mRNA. Overall, these studies provide a distinct mechanistic framework for the regulation of mutually exclusive splicing.

According to our pairing-mediated splicing model, alternative splicing includes two sequential processes: pairing and subsequent splicing (Fig. 10A), both of which could in- fluence the outcomes of alternative splicing. In the first process, the inclusion of an alternative exon could be modulated by pairing efficiency, which depends on the pairing strength, the interacting distance, the position of the alternative exon, and the proteins that facilitate long-range RNA interactions. Despite stochastic pairing, the stronger pairing interaction generally leads to more premRNA molecules that contain such pairing thereby increasing the frequency of targeted exon variant inclusion as evidenced by mutation and in vivo analysis (May et al. 2011; Yang et al. 2011). Moreover, the stronger pairing interaction might elicit more splicing repression via a looping-out mechanism (Jin et al. 2011), thus enhancing targeted exon variant inclusion. Notably, this inclusion was not determined by the upstream or downstream pairing strengths, but by the relative competitiveness between them (Fig. 10B). After pairing, the inclusion of an alternative exon is regulated by splicing speed and efficiency, which depend on the strength of the alternative exon splice site, the intronic distance from the selector element to the splice site, the presence of a splicing silencer/ enhancer, and the presence of activatorand repressor-binding proteins, as has been reported previously (Black 2003; Matlin et al. 2005; Blencowe 2006; Olson et al. 2007; Chen and Manley 2009; Nilsen and Graveley 2010; Fu and Ares 2014; Matera and Wang 2014). The splice site strength, in particular, which directly influences its recognition and splicing speed, is likely to play an important role in the pairing-mediated selection of an alternative exon. Pairing-mediated splicing is highly regulated by multiple mechanisms in an overlapping manner.

The conservation of the bidirectional RNA pairing interaction highlights the importance of its evolutionary and regulatory benefits. Bidirectional RNA pairings would be advantageous for the evolutionary expansion of exon clusters. Indeed, mimicking the expansion of either the downstreampairing-guided exon 4.1 or the upstream-pairing-guided exon 4.2 produced little conflict between splicing and regulatory (Fig. 7). In our previous model, the exon most proximal to the docking site would be included if the docking site assumes a linear conformation without specific RNA pairing interactions (Yang et al. 2011). However, this model could not explain how the duplicated exon would also be spliced in a 
mutually exclusive manner if this exon variant were further duplicated. The dual-docking-site model of this study could provide a reasonable explanation for its control mechanism in such an evolutionary expansion. Furthermore, any mutation in the single docking site would influence the interaction with the selector sequences and thus interfere with the inclusion of all within-cluster exon variants (Graveley 2005). We showed that mutation of either of the dual docking sites had little effect on srp exon 4 inclusion frequency (Fig. 1). This suggests that dual docking sites probably are much better able to tolerate this deleterious mutation. Finally, our results revealed that bidirectional RNA pairings may fine tune the inclusion of alternative exon. These bidirectional pairing interactions are not only characterized as competition but also cooperation. Such long-range RNA crosstalk, in cooperation with other elements, shapes a dynamic architectural network of complex alternative splicing regulation.

This unusual type of dynamic RNA secondary structures and architecture is not restricted to the srp gene. We showed that it also operates in Dscam exon 4 and 9 clusters. In addition, such dual secondary structures and architecture may be embedded in Drosophila RIC-3 (Supplemental Fig. S10). Some of them are young and clade-specific alternatively spliced exons (i.e., $\sim 80$ million years for $s r p$ ), whereas others are much more ancient and deeply conserved (i.e., $>300$ million years for Dscam exon 9 cluster). Although we have not found strong evidence for the arrangements of dual docking sites and their secondary structural interactions within Dscam exon 6 clusters, this possibility cannot be ruled out. Moreover, the unique docking site does not explain why its deletion did not result in a dramatic decrease in the inclusion frequency of all exons 6 (May et al. 2011). Higher-resolution comparative genomics from more species may allow determination of structurally conserved intronic sequences. In summary, the architectural landscape across arthropod species proposed here has shaped a potentially broad pattern for long-range RNA-RNA interaction in directing mutually exclusive splicing. Further investigations posed by this study involve exploring the extent and role of such complex RNARNA regulatory networks in other RNAs, particularly in explosively increasing long noncoding RNAs.

\section{MATERIALS AND METHODS}

\section{Materials}

Fruit flies (D. melanogaster), silkworms (B. mori), honeybees (A. mellifera) and other species were obtained as reported previously (Yang et al. 2011; Wang et al. 2012).

\section{Cloning and sequencing of srp and Dscam orthologs}

The sequences of the srp and Dscam genes in some insect species were obtained from FlyBase and NCBI (http://flybase.org/blast/ and http://blast.ncbi.nlm.nih.gov/blast). The genomic sequences of the Dscam genes for other insect species were determined by PCR and sequencing (Supplemental Table S1). Total RNA was isolated using TRIzol reagent (Life Technologies, Carlsbad, CA, USA) and was reverse transcribed using SuperScript III Reverse Transcriptase (Life Technologies) and an oligo $(\mathrm{dT})_{20}$ primer (Life Technologies). PCR was performed using a denaturing step at $95^{\circ} \mathrm{C}$ for 2 min and 35 cycles of a denaturing step at $95^{\circ} \mathrm{C}$ for $30 \mathrm{sec}$, an annealing step at $50^{\circ} \mathrm{C}-55^{\circ} \mathrm{C}$ for $30 \mathrm{sec}$ and an extension step at $72^{\circ} \mathrm{C}$ for $1 \mathrm{~min}$, followed by a final extension at $72^{\circ} \mathrm{C}$ for $10 \mathrm{~min}$. RT-PCR and PCR products were cloned into the pGEM-T Easy vector (Promega, Madison, WI, USA) for sequencing. The specific primers were designed based on the cDNA sequence (Supplemental Table S1). Total genomic DNA was extracted using the MiniBEST Universal Genomic DNA Extraction Kit (TaKaRa, Dalian, China). PCR cycling conditions were as follows: $95^{\circ} \mathrm{C}$ for $2 \mathrm{~min}$, followed by 35 cycles of $95^{\circ} \mathrm{C}$ for $30 \mathrm{sec}, 55^{\circ} \mathrm{C}-60^{\circ} \mathrm{C}$ for $30 \mathrm{sec}$, and $72^{\circ} \mathrm{C}$ for $2-4 \mathrm{~min}$, with a final extension at $72^{\circ} \mathrm{C}$ for $10 \mathrm{~min}$. Amplification products were cloned into the pGEM-T Easy vector for sequencing.

\section{Sequence alignments and RNA pairing predictions}

Alignment of specific regions among species was performed using the ClustalW2 software (http://www.ebi.ac.uk/clustalw/index.html). The RNA pairing interactions between the docking site and selector sequences were predicted using Mfold software (Zuker 2003). The RNA folding temperature was fixed at $37^{\circ} \mathrm{C}$, and the ionic conditions were $1 \mathrm{M} \mathrm{NaCl}$ without divalent ions. In some cases, the full sequences flanking the exon cluster were used as input for Mfold. In other cases, we only used the docking site, the selector, and their flanking sequences because of the limits of the Mfold program. The $5^{\prime}$ and $3^{\prime}$ splice site motif scores were calculated using the splice site predictor (range of 0 to 1 , with higher values predicting stronger splice sites) (Reese et al. 1997).

\section{Quantification of mRNA splice isoforms}

We assayed the ratio of srp and Dscam mRNA splice isoforms using RT-PCR and/or variant-specific restriction digestion analysis. Total RNA was isolated from different developmental stages, tissue samples and cells and was reverse transcribed using SuperScript III Reverse Transcriptase and gene-specific primers. Specific primers flanking the alternative exons were designed (Supplemental Table S1). PCR cycling conditions were as follows: $95^{\circ} \mathrm{C}$ for $2 \mathrm{~min}$, followed by 35 cycles of $95^{\circ} \mathrm{C}$ for $30 \mathrm{sec}, 55^{\circ} \mathrm{C}-60^{\circ} \mathrm{C}$ for $30 \mathrm{sec}$, and $72^{\circ} \mathrm{C}$ for $30-60 \mathrm{sec}$, with a final extension at $72^{\circ} \mathrm{C}$ for $10 \mathrm{~min}$. PCR products were then digested by exon-specific restriction enzymes. Images were captured using a CCD camera and mRNA isoforms were quantified by comparison of the integrated optical density of detected bands measured using the GIS 1D Gel Image System (ver. 3.73; Tanon, Shanghai, China). The error bars were calculated from the average of three independent experiments. The significance of differences was determined by the Student's $t$-test (two tails), and $\left(^{*}\right) P<0.05,\left(^{* *}\right) P<0.01$, and $\left(^{* * *}\right) P<0.001$ were deemed to indicate statistical significance.

\section{Minigene construction, mutagenesis, and transfection}

PCR was performed to amplify the DNA segments encompassing the upstream constitutive exon, the downstream constitutive exon 
and the intervening sequence for minigene constructs. Site mutagenesis was performed in both elements to disrupt the RNA stem according to the schematic diagrams of minigene constructs using primers (Supplemental Table S1). Disruptive and compensatory double mutations were performed to restore RNA stem structure, based on the schematic diagrams. To discriminate from the original exon 4.1 or 4.2 , point mutations were introduced to generate a specific restriction enzyme site. All constructs were confirmed by sequencing. WT and mutant constructs from D. melanogaster srp were inserted into the pMT/V5-HisA vector (Invitrogen, Carlsbad, CA, USA) under the metallothionein promoter. A. mellifera Dscam exon 4 was seldom seen in S2 cells transfected with the minigene constructs; therefore, we used BmN cells for transfection under control of the Baculovirus IE1 promoter (a gift from C. Gong, Shuzhou University, China). The plasmids were isolated using the Endo-free Plasmid Mini Kit I (OMEGA, Norcross, GA, USA) and transfected into Drosophila S2 cell lines or silkworm cells (BmN) using Lipofectamine 2000 (Life Technologies) according to the manufacturer's protocol. To induce the expression of the minigene, copper sulfate was added directly to the S2 cell culture medium to a final concentration of $500 \mu \mathrm{M}$. Total RNA was harvested $48 \mathrm{~h}$ after transfection using TRIzol reagent. RT-PCR and/or exon-specific restriction enzyme analysis were performed to detect the alternative splicing status. The values of the error bars used in this study were calculated from the average of three independent experiments.

\section{DATA DEPOSITION}

The Dscam gene sequences were deposited into GenBank with accession numbers KJ776429, KJ776436, and KJ776441.

\section{SUPPLEMENTAL MATERIAL}

Supplemental material is available for this article.

\section{ACKNOWLEDGMENTS}

This work was partly supported by research grants from the National Natural Science Foundation of China (31125011, 31430050, $31270844,31300655)$, the National Science and Technology Project (2012ZX09102301-009), the 973 Program (2014CB541700), and the Doctoral Foundation of the Ministry of Education (20110101130012).

Received July 15, 2015; accepted October 6, 2015.

\section{REFERENCES}

Anastassiou D, Liu HR, Varadan V. 2006. Variable window binding for mutually exclusive alternative splicing. Genome Biol 7: R2.

Baraniak AP, Lasda EL, Wagner EJ, Garcia-Blanco MA. 2003. A stem structure in fibroblast growth factor receptor 2 transcripts mediates cell-type-specific splicing by approximating intronic control elements. Mol Cell Biol 23: 9327-9337.

Black DL. 2003. Mechanisms of alternative pre-messenger RNA splicing. Annu Rev Biochem 72: 291-336.

Blackburn EH, Collins K. 2011. Telomerase: an RNP enzyme synthesizes DNA. Cold Spring Harb Perspect Biol 3: a003558.

Blencowe BJ. 2006. Alternative splicing: new insights from global analyses. Cell 126: 37-47.
Brierley I, Digard P, Inglis SC. 1989. Characterization of an efficient coronavirus ribosomal frameshifting signal: requirement for an RNA pseudoknot. Cell 57: 537-547.

Campbell K, Whissell G, Franch-Marro X, Batlle E, Casanova J. 2011. Specific GATA factors act as conserved inducers of an endodermal-EMT. Dev Cell 21: 1051-1061.

Chen M, Manley JL. 2009. Mechanisms of alternative splicing regulation: insights from molecular and genomics approaches. Nat Rev Mol Cell Biol 10: 741-754.

Chen BE, Kondo M, Garnier A, Watson FL, Püettmann-Holgado R, Lamar DR, Schmucker D. 2006. The molecular diversity of Dscam is functionally required for neuronal wiring specificity in Drosophila. Cell 125: 607-620.

Cruz JA, Westhof E. 2009. The dynamic landscapes of RNA architecture. Cell 136: 604-609.

Daines B, Wang H, Wang LG, Li YM, Han Y, Emmert D, Gelbart W, Wang X, Li W, Gibbs R, et al. 2011. The Drosophila melanogaster transcriptome by paired-end RNA sequencing. Genome Res 21: 315-324.

Dong Y, Cirimotich CM, Pike A, Chandra R, Dimopoulos G. 2012. Anopheles NF- $\kappa B$-regulated splicing factors direct pathogen-specific repertoires of the hypervariable pattern recognition receptor AgDscam. Cell Host Microbe 12: 521-530.

Fu XD, Ares M. 2014. Context-dependent control of alternative splicing by RNA-binding proteins. Nat Rev Genet 15: 689-701.

Graveley BR. 2005. Mutually exclusive splicing of the insect Dscam premRNA directed by competing intronic RNA secondary structures. Cell 123: 65-73.

Hattori D, Demir E, Kim HW, Viragh E, Zipursky SL, Dickson BJ. 2007. Dscam diversity is essential for neuronal wiring and self-recognition. Nature 449: 223-227.

Hattori D, Chen Y, Matthews BJ, Salwinski L, Sabatti C, Grueber WB, Zipursky SL. 2009. Robust discrimination between self and nonself neurites requires thousands of Dscam1 isoforms. Nature 461: 644-648.

He HH, Kise Y, Izadifar A, Urwyler O, Ayaz D, Parthasarthy A, Yan B, Erfurth ML, Dascenco D, Schmucker D. 2014. Cell-intrinsic requirement of Dscam1 isoform diversity for axon collateral formation. Science 344: 1182-1186.

Howe KJ, Ares M Jr. 1997. Intron self-complementarity enforces exon inclusion in a yeast pre-mRNA. Proc Natl Acad Sci 94: 12467-12472.

Jin YF, Yang Y, Zhang P. 2011. New insights into RNA secondary structure in the alternative splicing of pre-mRNAs. RNA Biol 8: 450-457.

Li F, Zheng Q, Ryvkin P, Dragomir I, Desai Y, Aiyer S, Valladares O, Yang JM, Bambina S, Sabin LR, et al. 2012. Global analysis of RNA secondary structure in two metazoans. Cell Rep 1: 69-82.

Matera AG, Wang ZF. 2014. A day in the life of the spliceosome. Nat Rev Mol Cell Biol 15: 108-121.

Matlin AJ, Clark F, Smith CWJ. 2005. Understanding alternative splicing: towards a cellular code. Nat Rev Mol Cell Biol 6: 386-398.

Matthews BJ, Kim ME, Flanagan JJ, Hattori D, Clemens JC, Zipursky SL, Grueber WB. 2007. Dendrite self-avoidance is controlled by Dscam. Cell 129: 593-604.

May GE, Olson S, McManus CJ, Graveley BR. 2011. Competing RNA secondary structures are required for mutually exclusive splicing of the Dscam exon 6 cluster. RNA 17: 222-229.

Miura SK, Martins A, Zhang KX, Graveley BR, Zipursky SL. 2013. Probabilistic splicing of Dscam 1 establishes identity at the level of single neurons. Cell 155: 1166-1177.

Muh SJ, Hovhannisyan RH, Carstens RP. 2002. A non-sequence-specific double-stranded RNA structural element regulates splicing of two mutually exclusive exons of fibroblast growth factor receptor 2 (FGFR2). J Biol Chem 277: 50143-50154.

Nilsen TW, Graveley BR. 2010. Expansion of the eukaryotic proteome by alternative splicing. Nature 463: 457-463.

Olson S, Blanchette M, Park J, Savva Y, Yeo GW, Yeakley JM, Rio DC, Graveley BR. 2007. A regulator of Dscam mutually exclusive splicing fidelity. Nat Struct Mol Biol 14: 1134-1140. 
Yue et al.

Pan Q, Shai O, Lee LJ, Frey J, Blencowe BJ. 2008. Deep surveying of alternative splicing complexity in the human transcriptome by highthroughput sequencing. Nat Genet 40: 1413-1415.

Patient RK, McGhee JD. 2002. The GATA family (vertebrates and invertebrates). Curr Opin Genet Dev 12: 416-422.

Pervouchine DD. 2014. IRBIS: a systematic search for conserved complementarity. RNA 20: 1519-1531.

Petersen UM, Kadalayil L, Rehorn KP, Hoshizaki DK, Reuter R, Engstrom Y. 1999. Serpent regulates Drosophila immunity genes in the larval fat body through an essential GATA motif. EMBO J 18: 4013-4022.

Plocik AM, Graveley BR. 2013. New insights from existing sequence data: generating breakthroughs without a pipette. Mol Cell 49: 605-617.

Raker VA, Mironov AA, Gelfand MS, Pervouchine DD. 2009. Modulation of alternative splicing by long-range RNA structures in Drosophila. Nucleic Acids Res 37: 4533-4544.

Reese MG, Eeckman FH, Kulp D, Haussler D. 1997. Improved splice site detection in Genie. J Comput Biol 4: 311-323.

Schmucker D, Clemens JC, Shu H, Worby CA, Xiao J, Muda M, Dixon JE, Zipursky SL. 2000. Drosophila Dscam is an axon guidance receptor exhibiting extraordinary molecular diversity. Cell 101: 671-684.

Senger K, Harris K, Levine M. 2006. GATA factors participate in tissuespecific immune responses in Drosophila larvae. Proc Natl Acad Sci 103: $15957-15962$.

Smith CWJ. 2005. Alternative splicing-when two's a crowd. Cell 123: $1-3$.

Sultan M, Schulz MH, Richard H, Magen A, Klingenhoff A, Scherf M, Seifert M, Borodina T, Soldatov A, Parkhomchuk D, et al. 2008. A global view of gene activity and alternative splicing by deep sequencing of the human transcriptome. Science 321: 956-960.

Tingvall TO, Roos E, Engström Y. 2001. The GATA factor Serpent is required for the onset of the humoral immune response in Drosophila embryos. Proc Natl Acad Sci 98: 3884-3888.

Waltzer L, Bataillé L, Peyrefitte S, Haenlin M. 2002. Two isoforms of Serpent containing either one or two GATA zinc fingers have different roles in Drosophila haematopoiesis. EMBO J 21: 54775486.

Wang XB, Li GL, Yang Y, Wang WF, Zhang WJ, Pan HW, Zhang P, Yue Y, Lin H, Liu BP, et al. 2012. An RNA architectural locus control region involved in Dscam mutually exclusive splicing. Nat Commun 3: 1255.

Watson FL, Puttmann-Holgado R, Thomas F, Lamar DL, Hughes M, Kondo M, Rebel VI, Schmucker D. 2005. Extensive diversity of Igsuperfamily proteins in the immune system of insects. Science 309: 1874-1878.

Watthanasurorot A, Jiravanichpaisal P, Liu HP, Söderhäll I, Söderhäll K. 2011. Bacteria-induced Dscam isoforms of the crustacean, Pacifastacus leniusculus. PLoS Pathog 7: e1002062.

Yang Y, Zhan LL, Zhang WJ, Sun F, Wang WF, Tian N, Bi JP, Wang HT, Shi DK, Jiang YJ, et al. 2011. RNA secondary structure in mutually exclusive splicing. Nat Struct Mol Biol 18: 159-168.

Zhan XL, Clemens JC, Neves G, Hattori D, Flanagan JJ, Hummel T, Vasconcelos ML, Chess A, Zipursky SL. 2004. Analysis of Dscam diversity in regulating axon guidance in Drosophila mushroom bodies. Neuron 43: 673-686.

Zuker M. 2003. Mfold web server for nucleic acid folding and hybridization prediction. Nucleic Acids Res 31: 3406-3415. 
RNA 21: 1219-1232 (2015)

\section{Corrigendum: Conserved RNA secondary structures and long-range interactions in hepatitis $C$ viruses \\ MARKUS FRICKE, NADIA DÜNNES, MARGARITA ZAYAS, RALF BARTENSCHLAGER, MICHAEL NIEPMANN, and MANJA MARZ}

In the above-noted article, the Acknowledgments section was incomplete and should instead read as follows:

"This work was in part funded by Deutsche Forschungsgemeinschaft (DFG) projects MA-5082/1, NI-604/2-2, SPP 1596, IRTG 1384, SFB 1021, and the Carl-Zeiss-Stiftung."

This article has been corrected in both the PDF and full-text HTML files online.

doi: $10.1261 /$ rna.058123.116

RNA 22: 96-110 (2016)

\section{Corrigendum: Long-range RNA pairings contribute to mutually exclusive splicing YUAN YUE, YUN YANG, LANZHI DAI, GUOZHENG CAO, RAN CHEN, WEILING HONG, BAOPING LIU, YANG SHI, YIJUN MENG, FENG SHI, MU XIAO, and YONGFENG JIN}

In the Discussion section (first paragraph, third sentence) on page 105 of the above-noted article, the sentence "The RNA architectures identified in our study were not predicted by in silico approaches (Pervouchine 2014), nor were they detected using recent high-throughput RNA structure sequencing techniques (Daines et al. 2011; Li et al. 2012)" was not clear and should instead read as follows: "The RNA bidirectional structural architectures identified in our study were not detected using recent high-throughput RNA structure sequencing techniques (Daines et al. 2011; Li et al. 2012), nor were they discovered by in silico approaches, although stem II of the Drosophila srp pre-mRNA was predicted (Raker et al. 2009; Pervouchine 2014).”

Accordingly, the following reference should be included in the References section:

Raker VA, Mironov AA, Gelfand MS, Pervouchine DD. 2009. Modulation of alternative splicing by long-range RNA structures in Drosophila. Nucleic Acids Res 37: 4533-4544.

The authors apologize for any confusion this may have caused.

This article has been corrected in both the PDF and full-text HTML files online.

doi: $10.1261 /$ rna.058008.116 

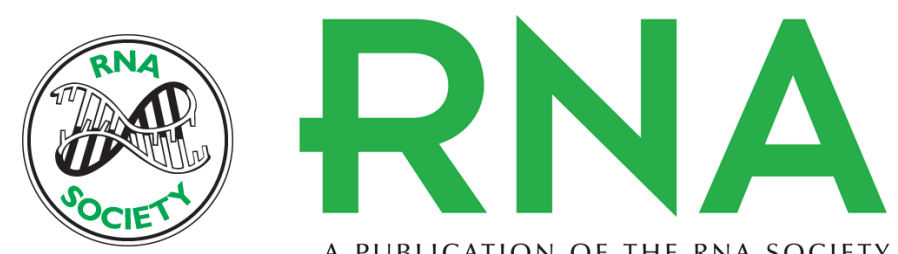

A PUBLICATION OF THE RNA SOCIETY

\section{Long-range RNA pairings contribute to mutually exclusive splicing}

Yuan Yue, Yun Yang, Lanzhi Dai, et al.

RNA 2016 22: 96-110 originally published online November 9, 2015

Access the most recent version at doi:10.1261/rna.053314.115

\section{Supplemental http://rnajournal.cshlp.org/content/suppl/2015/11/04/rna.053314.115.DC1 \\ Material}

\section{Related Content}

References

Creative Commons License
Email Alerting Service

Corrigendum: Long-range RNA pairings contribute to mutually exclusive splicing Yuan Yue, Yun Yang, Lanzhi Dai, et al. RNA October , 2016 22: 1640.2

This article cites 46 articles, 14 of which can be accessed free at: http://rnajournal.cshlp.org/content/22/1/96.full.html\#ref-list-1

Articles cited in: http://rnajournal.cshlp.org/content/22/1/96.full.html\#related-urls

This article is distributed exclusively by the RNA Society for the first 12 months after the full-issue publication date (see http://rnajournal.cshlp.org/site/misc/terms.xhtml). After 12 months, it is available under a Creative Commons License (Attribution-NonCommercial 4.0 International), as described at http://creativecommons.org/licenses/by-nc/4.0/.

Receive free email alerts when new articles cite this article - sign up in the box at the top right corner of the article or click here.

To subscribe to $R N A$ go to:

http://rnajournal.cshlp.org/subscriptions 